\title{
Near Infrared Diode Laser THz Systems
}

\author{
Carsten Brenner $^{1}$, Yinghui Hu ${ }^{1}$, Jared Gwaro ${ }^{1}$, Nils Surkamp ${ }^{1}$, Benjamin Döpke ${ }^{1}$, Martin R. Hofmann ${ }^{1}$, \\ Besher Kani ${ }^{2}$, Andreas Stöhr ${ }^{2}$, Bernd Sumpf ${ }^{3}$, Andreas Klehr ${ }^{3}$, and Jörg Fricke ${ }^{3}$ \\ ${ }^{1}$ Photonics and Terahertz Technology, Ruhr-Universität Bochum, 44801 Bochum, Germany \\ ${ }^{2}$ ZHO/Optoelektronik, Universität Duisburg-Essen, 47048 Duisburg, Germany \\ ${ }^{3}$ Ferdinand-Braun-Institut, Leibniz-Institut für Höchstfrequenztechnik, 12489 Berlin, Germany
}

Correspondence: Carsten Brenner (carsten.brenner@ ruhr-uni-bochum.de)

Received: 28 January 2018 - Revised: 30 March 2018 - Accepted: 5 April 2018 - Published: 4 September 2018

\begin{abstract}
The generation and detection of radiation in the $\mathrm{THz}$ frequency range can be achieved with many different electronic and photonic concepts. Among the many different photonic $\mathrm{THz}$ systems the most versatile are based on diode lasers. In this paper we describe and review the different concepts and optimization ideas for diode laser based THz systems in order to achieve the best performance for different types of $\mathrm{THz}$ setups.
\end{abstract}

\section{Introduction}

Photonic approaches to generate and detect $\mathrm{THz}$ frequencies with diode lasers can be subdivided in frequency domain (FD) and time domain (TD) approaches. FD systems usually emit and detect a single frequency over time while TD systems are based on ultrashort pulses and sample a time transient point by point. The contributing frequencies of a TD system are then resolved by a Fourier transform. The classical configuration of those systems is described in Sect. 2.

Originally diode laser based $\mathrm{THz}$ systems have been used especially in FD setups. But diode lasers have approached specifications that enable typical TD applications in the last decade. In this paper we will give a brief review about the different approaches of diode laser use for FD systems as well as TD systems.

While the first photonic systems for difference frequency generation in the $\mathrm{THz}$ range were implemented with solid state lasers (Brown et al., 1993; McIntosh et al., 1995) and Bolometers, it was clear that diode lasers can fulfill this task in a more cost-effective way with more compact setups. Consequently the solid state laser systems were replaced by diode laser systems and with the use of self-mixing techniques the road was paved for the next steps (Verghese et al., 1998).

In contrast to TD systems the inherent advantage of a FD system is given by the achievable frequency resolution in the $\mathrm{THz}$ range and the accessible power in a certain bandwidth. Although in TD systems the accessible bandwidth in a given amount of time is usually larger, the two before mentioned advantages are especially important for spectroscopic applications where a high frequency resolution is beneficial (Verghese et al., 1998; Hübers et al., 2011).

To achieve such a high frequency solution, there was a constant optimization process for FD systems in the following years. The main goals were given by decreasing the laser linewidth, the size of the systems or improving the noise characteristics of the overall system. Several approaches were realized to achieve these goals:

a. The use of stabilized laser systems (see Sect. 3.1).

b. The use of two color lasers which should result in a decrease of noise at the difference frequency (see Sect. 3.1.2).

c. The monolithic integration of a two-color laser system represents the most compact and cost-effective pump laser source. But such systems usually suffer from a low tunability in comparison to external cavity approaches or stand-alone single frequency devices (see Sect. 3.1.2).

Parallel to the optimization of the noise and linewidth features other approaches of multi-frequency $\mathrm{THz}$ generation were investigated (Morikawa et al., 1999, 2004; Gregory et al., 2005; Tani et al., 2005; Shibuya et al., 2006). By using more than two lasers or multimode diode lasers, 
there is the option to detect more than one difference frequency at the same time. The higher the number of the optical modes which are emitted at the same time the higher the number of the resulting $\mathrm{THz}$ modes. By using cost-effective multimode lasers these techniques can ultimately be used in the same way as time domain spectroscopy (TDS) systems based on ultrashort pulses. Therefore this concept is called Quasi-TDS. The main drawback of QTDS is a lower SNR in the resulting frequency spectrum (Scheller and Koch, 2009; Kohlhaas et al., 2017). When using external cavity lasers it is possible to realize a system with switchable two color- and QTDS operation (Brenner et al., 2010) (see Sect. 3.2).

Another field of research was the change of the wavelength region of the pumping lasers to $1550 \mathrm{~nm}$ (Sartorius et al., 2012; Fice et al., 2010; Ryu et al., 2012; Rymanov et al., 2013; Göbel et al., 2013; Hisatake et al., 2014) or $1 \mu \mathrm{m}$ (Kitahara et al., 2013; Tanabe et al., 2009) and $1.3 \mu \mathrm{m}$ (Moon et al., 2014). The development of new photomixers based on telecommunication diode mixers shifted the focus to diode laser systems in the telecommunication range which makes industry components as laser sources easily accessible.

During most of the research one of the main issues was the fact that nearly any kind of noise (e.g. noise in frequency, phase, amplitude) shows up as intensity noise due to the interferometric nature of the typical self-mixing setup (Verghese et al., 1998). To distinguish amplitude and phase effects in such a system a mechanical delay stage was state of the art until tunable lasers were used to solve that problem (Demers et al., 2008; Roggenbuck et al., 2010; Stanze et al., 2011). Following these approaches many different modulation schemes have been shown to get rid of this mechanical part of the setup (Hisatake et al., 2014; Song et al., 2017) (see Sect. 3.3).

Furthermore FD systems can be used in a combination with electronic setups which make enable use diode lasers based systems as local oscillators in vector network analyzers (Friederich et al., 2010; Rouvalis et al., 2011) or to achieve high data rates in telecommunication systems (Rymanov et al., 2013).

In contrast to FD systems the problems in TD systems have a slightly different focus. The main challenges for diode laser TD systems are given by:

a. The reduction of the pulse width in external cavity lasers in order to achieve a reasonably high $\mathrm{THz}$ bandwidth (see Sect. 4.1).

b. The use of monolithic approaches to generate $\mathrm{THz}$ frequency by short cavities and/or high harmonics of operation (see Sect. 4.2).

c. The use of different techniques to remove the delay line (see Sect. 4.3).

\section{Classical systems}

\subsection{Classical frequency domain system}

The classical realisation of a frequency domain (FD) system is done with two lasers, at least one beamsplitter, a mechanical delay stage and two photomixers as transmitter (Tx) and receiver $(\mathrm{Rx})$ as depicted in the left part of Fig. 1. The transmitting photomixer converts the signal from optical frequencies to $\mathrm{THz}$ frequencies which can be done in many different configurations (Preu et al., 2015). The receiver generates typically a DC photocurrent due to the self mixing of the $\mathrm{THz}$ signal with the initial optical signal.

To maximize the signal at the receiver the lengths of the transmitter path and receiver path should be identical. The optical path difference (OPD) is zero at that position. An OPD which is smaller than the coherence length of the used lasers is sufficient to generate a signal, though. Unfortunately any amplitude or phase influence of a device under test has an impact on the measurement signal. Moreover, the delay stage which shifts the signal around OPD $=0$ is necessary to distinguish between amplitude and phase changes in a classical FD system.

\subsection{Classical time domain system}

The classical realisation of a time domain system is depicted schematically in the right part of Fig. 1. Instead of two lasers with narrowband linewidths a modelocked laser is used to generate ultrashort pulses. The optical pulse train is split into two parts. One is directed to the transmitter Tx which generates a $\mathrm{THz}$ transient that is sent to the device under test (DUT). The Tx may be realized either based on a photoconductive switch or a nonlinear crystal. The other part of the pulse train passes a variable delay stage and is then coupled onto the receiver $\mathrm{Rx}$ where the received $\mathrm{THz}$ field is sampled. The detection itself can be based on nonlinear crystals or photoconducting switches (Jepsen et al., 2011).

Like in a FD system a mechanical delay stage is usually used to sample the $\mathrm{THz}$ transient over a certain time. The measured frequency components are then retrieved by a Fourier transform of the acquired transient. The achievable resolution is consequently limited by the total length of the scanned delay. To beat the resolution of a FD system, though, the length of that delay stage would have to be in the range of the coherence length of the low linewidth lasers in the FD system. This is not a practical solution. Moreover the repetition rate of a ultrashort pulse laser will prevent any measurement at narrowband resonances which might be placed in between two cavity modes.

\section{FD systems}

In the following section we will give an overview about different concepts to implement diode lasers in FD systems and 


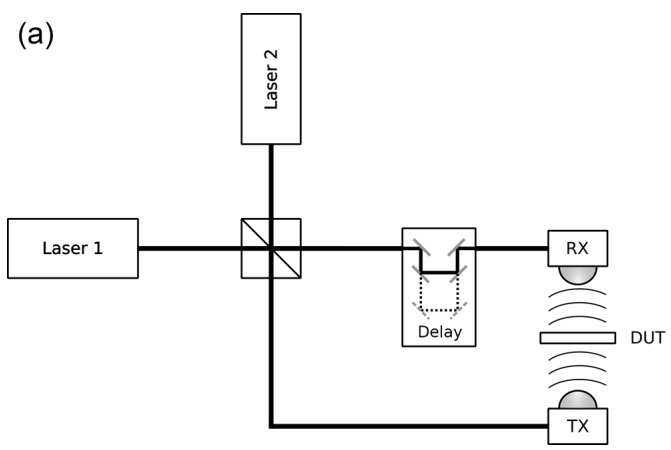

(b)

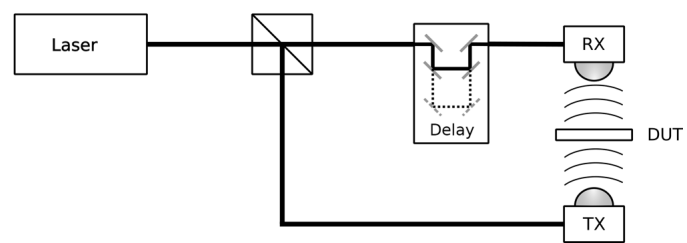

Figure 1. (a) Classical FD system with two lasers, delay stage, and two photomixers for transmission (Tx) and detection (Rx). (b) Classical TD system with ultrashort pulse laser and delay stage.

to improve their performance. One of the main distinctions is the use of monolithic diode lasers versus the use of external cavity lasers. Monolithic lasers include all necessary components in a single semiconductor chip. This makes the design process very demanding but results in very compact and costeffective devices. In an external cavity lasers the diode is only part of a bigger laser cavity. This increases the complexity of the system but allows for very high flexibility and typically results in superior performance.

\subsection{Increasing the frequency resolution}

While the first FD systems were based on $\mathrm{Ti}: \mathrm{Sa}$ lasers (Brown et al., 1993) the second generation of difference frequency generation systems was already based on diode lasers (McIntosh et al., 1995; Chen et al., 1997). The main advantages are compactness, cost-effectiveness and ease of use. The biggest advantage of the FD systems itself in comparison with THz TDS systems is the capability to achieve a much higher frequency resolution. This resolution increase is based on the fact that in a FD system the linewidth of the optical components determines the frequency resolution rather than the properties of a mechanical delay stage.

To even strengthen this advantage of FD systems there are two options. Either one may use two stabilized lasers with low linewidths or two-color laser systems. The resolution increase of the first approach can directly be seen based on the linewidth argument. The advantage of the latter one is given by the reduction in noise of the generated $\mathrm{THz}$ frequency as the optical noise components are removed by the homodyne mixing process.

\subsubsection{Stabilizing the lasers}

Over the first decade of the use of diode lasers for FD systems the systems achieved smaller linewidth over time. Here we give just a few exemplary setups to show the progress: The first approach was based on distributed Bragg reflector (DBR) lasers without any stabilization scheme (McIntosh et al., 1995). A few years later this was superseded by ex- ternal cavity lasers which provided a much smaller optical linewidth and therefore higher resolution in the $\mathrm{THz}$ range (Matsuura et al., 1998; Chen et al., 1997).

By using DFB lasers, 10 years later, it was possible to achieve very simple and compact optical pumping schemes (Göbel et al., 2008). By implementing external control loops even linewidths in the MHz range became possible (Demers et al., 2008; Deninger et al., 2008). The originally proposed compact, and cost-effective use of FD systems has become possible when photodiodes for $\mathrm{THz}$ generation in the range of $1550 \mathrm{~nm}$ became available (Preu et al., 2015). This introduced the chance to use readily available commercial DFB lasers from the telecommunication industry and showed similar results with "off-the-shelf butterfly packages" (Stanze et al., 2011).

\subsubsection{Two-color lasers}

Another approach to achieve a compact FD THz system is based on two-color laser sources. The first practical advantage is the inherent superposition of the two beams in contrast to a meticulous alignment procedure in the former two laser approach. But regarding the noise characteristic of a THz FD system the fundamental advantage of lasers which emit two colors simultaneously is based on the self mixing procedure of the $\mathrm{THz}$ generation. As all noise contributions, which are present in both wavelengths, are removed during the mixing process the resulting $\mathrm{THz}$ linewidth may be smaller than the optical linewidth of the pump laser source. As two-color lasers use a shared cavity for both laser modes the noise on both colors is suppressed in the THz generation process (Tani et al., 2000, 2005).

To accomplish this two mode operation either external cavity configurations can be used (Wang and Pan, 1994; Friedrich et al., 2008; Park et al., 2008; Chi et al., 2011; Paquet et al., 2016) but also external filtering with an etalon or electronic filtering can be applied (Naftaly et al., 2005; Criado et al., 2012). Depending on the setup, the characteristics of the output might have several advantages or disadvantages. When using an external cavity configuration usu- 

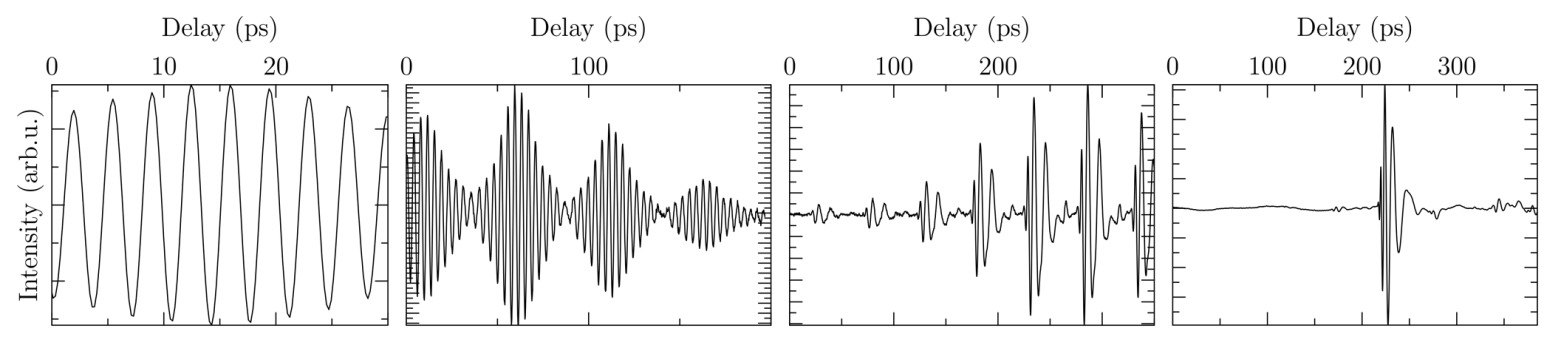

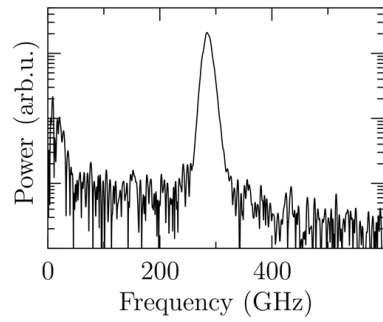

(a)

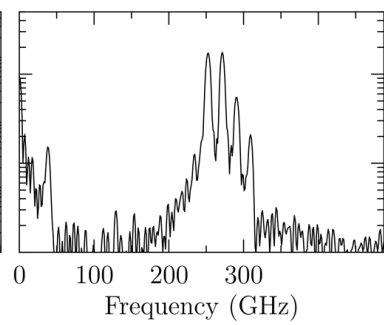

(b)

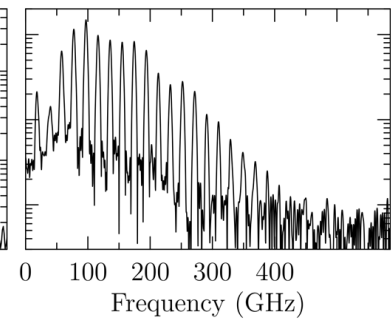

(c)

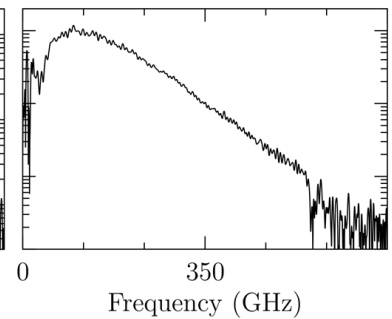

(d)

Figure 2. Measured THz transients and spectra for different continuous wave laser configurations. (a) Two color laser. (b) Multimode laser with low number of optical modes. (c) Multimode laser with round trip frequency of approximately $20 \mathrm{GHz}$. (d) External cavity multimode laser with lower round trip frequency of approximately $300 \mathrm{MHz}$.

ally a strong frequency filtering makes a continuous tuning of the difference frequency difficult as the mode competition is complex. Consequently a change in the difference frequency might have a big influence on the power ratio of the two laser modes. Unfortunately a power ratio different from 1 makes the mixing process less efficient. Using a feedback which is less frequency selective increases the linewidth but enables even long measurements for imaging applications (KleineOstmann et al., 2001; Matus et al., 2004).

Even better stability and noise reduction can be accomplished when the two-color laser is monolithically integrated. Over the last decade several geometries of monolithic distributed feedback lasers DFB and DBR implementations were realized and tested (Klehr et al., 2008; Osborne et al., 2008; O'Brien et al., 2010; Ryu et al., 2012; Göbel et al., 2012; Theurer et al., 2013; Gwaro et al., 2017). The biggest drawback is typically the limited tuning range. This is usually smaller than in other approaches. Values up to $800 \mathrm{GHz}$ as difference frequency were achieved (Moon et al., 2014) in contrast to $30 \mathrm{THz}$ achieved with external cavity lasers (Hoffmann et al., 2006).

\subsection{Multi-frequency systems}

When frequency resolution is not the main requirement, it is possible to measure several frequencies at the same time by using lasers which are running in multimode operation (Morikawa et al., 1999, 2004; Gregory et al., 2005; Tani et al., 2005; Shibuya et al., 2006). By using several optical modes, the number of $\mathrm{THz}$ frequencies increases with the square of the number of optical modes as all mixing products appear in the output. If the number of optical modes is $n$, the total number of generated difference frequencies is $n(n-1) / 2$. Figure 2 shows measured time transients and frequency spectra with different numbers of generated $\mathrm{THz}$ modes.

This scheme works with the cheapest multimode diode lasers available as there are three major effects which prevent the signal from being lost due to noise.

a. The optical phase noise is removed in the homodyne detection scheme as long as the path difference between emitter and receiver arm is not too large. Therefore the detected current at the receiver is not strongly affected by optical phase noise even without any optical phase locking between the different modes.

b. Intensity fluctuations due to mode competition can be easily removed by a slow detector or an additional averaging process. Therefore the average output of the DC current at the receiver (see Sect. 2.1) will show the mixing result of the "average" optical spectrum.

c. A superposition of arbitrary frequencies would result in a chaotic output but the frequency spacing of the multimode diode lasers is well determined by the cavity length. Therefore the superposition of all difference frequencies results in a constructive addition of the signal components.

For measurement purposes the resulting $\mathrm{THz}$ spectrum can be treated like a TD spectroscopy signal. Therefore this operation regime is called quasi time domain spectroscopy (QTDS) (Scheller and Koch, 2009; Kohlhaas et al., 2017). The implementation of a QTDS system can be done with 
very cost-effective optical components (Probst et al., 2015). The trade-off in comparison to standard TDS systems is a reduced SNR in the resulting spectrum and an usually quite large modespacing in the range of $10 \mathrm{~s}$ of $\mathrm{GHz}$ (see Fig. 2c).

To reduce the modespacing in the $\mathrm{THz}$ spectrum and to gain more control over the resulting $\mathrm{THz}$ spectrum, an external cavity diode laser configuration can be used. The longer cavity length leads to a typical mode spacing in the range of below $1 \mathrm{GHz}$. Furthermore the system enables to choose between either two color mode operation or QTDS operation (see Fig. 2a and c) (Brenner et al., 2010).

The realization of a multimode system with three separate diode lasers on the other hand enables to build a self referencing system. Two out of the three resulting difference frequencies will change when one laser mode is tuned to another frequency. As the third difference frequency is constant, the phase of all three frequencies can be calculated out of the resulting transient (Thirunavukkuarasu et al., 2015).

\subsection{Replacement of the moveable delay line}

One of the biggest obstacles for an easy and robust implementation of any $\mathrm{THz}$ system has been the requirement of a mechanical delay stage. To determine amplitude and phase changes in a device under test, usually the photocurrent at the receiver antenna is recorded by changing the position of the delay stage around OPD $=0$ (see Sect. 2). Using a position different than $\mathrm{OPD}=0$ opens up new possibilities and makes it possible to remove the delay stage completely.

The signal of different frequencies will show a maximum photocurrent at the receiver at different OPD. Consequently a frequency scan at a position OPD $\neq 0$ will result in a modulation of the measured signal. Roggenbuck et al. (2010) called this a "scan of an interference pattern in frequency". Many different modulation schemes have been realised which either work with a frequency scan or with a phase modulation in the optical regime (Demers et al., 2008; Roggenbuck et al., 2010; Stanze et al., 2011; Hisatake et al., 2014; Song et al., 2017).

The requirement for a delay stage is then replaced by the interplay of OPD, overall frequency tuning range, and tuning resolution. Increasing the OPD will increase the modulation frequency and can only be used if the tuning step size is small enough to resolve the frequency of the interference pattern. The overall tuning range on the other hand will determine the length of the measurable period of the interference pattern and therefore limits the preciseness of the measurement. The analogon in microwave technology is a frequency modulated continuous wave radar (FMCW).

\subsection{Photonic local oscillators}

In recent years diode laser based $\mathrm{THz}$ sources were also used as oscillators for data transmission with $\mathrm{THz}$ carrier frequencies and local oscillators in vector network analyzers. As electronic oscillators have to be multiplied to achieve frequencies in the range of several $100 \mathrm{GHz}$, the overall performance of those devices usually degrades. Photonic systems, on the other hand, can achieve very high $Q$ values in the first place and relevant characteristics for oscillators are improved when mixing down to lower frequencies (Friederich et al., 2010; Rouvalis et al., 2011). Moreover, the use of photonic oscillators makes it possible to achieve very high data rates due to the achievable carrier frequencies. Up to $59 \mathrm{Gbit} \mathrm{s}^{-1}$ in a $10 \mathrm{GHz}$ bandwidth at a carrier frequency of $325 \mathrm{GHz}$ were achieved recently (Hermelo et al., 2017).

\section{TD systems}

For many years TD $\mathrm{THz}$ system were the realm of solid state lasers as only those systems provided pulses which were short enough and had enough power to generate $\mathrm{THz}$ pulses. With the advance of technology, fiber lasers were used as typical laser source as the parameters are usually less outstanding but sufficient to generate $\mathrm{THz}$ pulses. Diode lasers, on the other hand, had a difficult start as their longer pulses reduced the achievable $\mathrm{THz}$ bandwidth. Also the power they provide is not as high as in solid state laser systems but a so called master oscillator power amplifier combination can be used to boost the power to application relevant levels. Beside these crucial parameters, there are several other goals which are actively researched with diode laser based systems to increase the performance in TD setups.

\subsection{External cavity diode lasers}

As stated above, one of the main issues in TD systems is the achievable bandwidth which depends on the pulse duration of the optical pulse. Most diode laser systems provide longer pulses than solid state or fiber lasers which reduces the overall accessible bandwidth of the THz system. Nonetheless current records are in the range of $200 \mathrm{fs}$ in different geometries of external cavity diode laser systems (Döpke et al., 2015). Although TD systems can be realized also with longer pulse duration, shorter pulses give more headroom for system optimization.

The challenge to generate high power pulses with durations in the range of $\ll 1 \mathrm{ps}$ with semiconductor lasers is based on the complex interplay of the carrier dynamics and the ultrashort pulse. A successful realisation is typically based on an oscillator with succeeding amplifier and compressor. The main task for the oscillator is then to generate pulses with a large optical bandwidth. The pulses are usually chirped so that they can be amplified without strong distortions and compressed afterwards to bandwidth limited sub-ps pulses with sufficient power to drive $\mathrm{THz}$ photomixers (Jördens et al., 2008). 


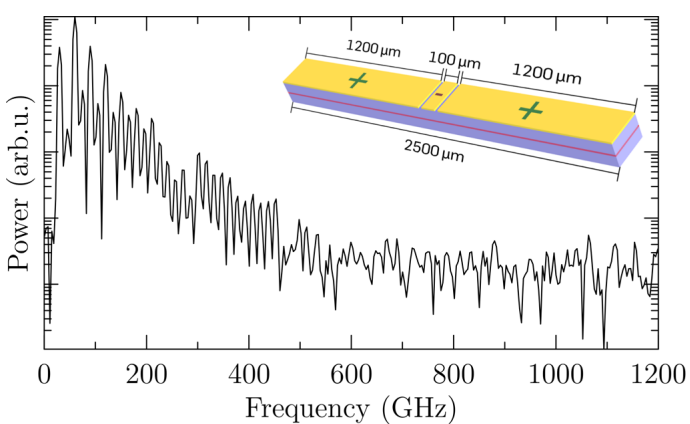

(a)

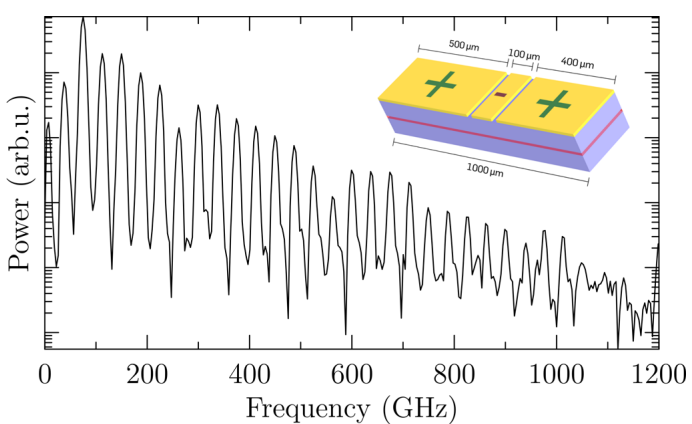

(b)

Figure 3. Measured THz frequency spectra of different monolithic modelocked diode lasers in a TD THz setup. The length of the diode is the dominant effect for the mode spacing. (a) Diode of $2.5 \mathrm{~mm}$ length operating in colliding pulse regime. (b) Diode of $1 \mathrm{~mm}$ length operating in a self pulsation regime.

Due to these facts external cavity laser systems are still rather sophisticated and the realisation of monolithic short pulse systems is another research topic with a huge potential.

\subsection{Monolithic modelocked lasers}

When bandwidth is not the main issue but compactness and simplicity, monolithic modelocked lasers are a good option to replace more sophisticated systems. As well as in FD systems these diodes are then the smallest option which emit short pulses directly out of the device. Although the pulse length is typically still in the range of $\approx 1 \mathrm{ps}$, it is possible to generate frequencies up to $1 \mathrm{THz}$ by photomixing. The dominant influence for the mode spacing in the resulting $\mathrm{THz}$ spectrum is the repetition rate of the laser which is determined by the length of the diode as can be seen in Fig. 3 .

The simplest THz TDS system is based on self modelocked Fabry Perot lasers in combination with a dispersive fiber of the correct length for chirp compensation. Such a system could be used for frequency generation up to $600 \mathrm{GHz}$ (Sato, 2003; Merghem et al., 2017). It is also possible to use modelocked lasers to realise FD systems by filtering out the beating of two cavity modes (Criado et al., 2012).

\subsection{Advanced scanning schemes}

The use of a mechanical delay line poses many problems in TD systems as well as in FD systems. During the last decade at least 3 different approaches were investigated using solid state lasers or fiber lasers. Först et al. (2005) used two frequency locked ultrashort pulse lasers with slightly different repetition rate for generating and detecting $\mathrm{THz}$ pulses. This so called ASOPS (asynchronous optical sampling) scheme enables very fast scanning rates with the drawback that the relevant pulse data is acquired with a rather low efficiency. Hochrein et al. (2010) implemented another scanning scheme by using a long fixed delay line. By changing the repetition rate of the laser the $\mathrm{THz}$ transient can be sampled. This scheme is called OSCAT (optical scanning by cavity tuning). Tauser et al. (2008) and Kim and Yee (2010) improved the ASOPS scheme by controlling the change of the repetition rate and called this ECOPS (electronically controlled optical sampling). This makes it possible to scan the $\mathrm{THz}$ pulse with a higher efficiency. These setups are usually very demanding since a precise frequency locking of the lasers is necessary and OSCAT and ECOPS suffer from the drawback that a continuous change inside the laser cavity is mandatory to achieve the scanning. This rises new problems concerning stability and noise. In principle these concepts can also be realized with diode laser systems but their timing jitter is typically higher which poses the biggest problem for the OSCAT approach.

\section{Conclusion}

Over the last 3 decades a huge zoo of different $\mathrm{THz}$ setups has been developed. Due to their versatility and increasing performance, diode lasers have played a major role in the development of $\mathrm{THz}$ systems in the past, and they will certainly do so in the future. Most often, diode lasers are able to replace other more expensive and complex lasers as their key parameters can be perfectly shaped to fit to the application.

Data availability. The datasets are available from the corresponding author on reasonable request.

Competing interests. The authors declare that they have no conflict of interest.

Special issue statement. This article is part of the special issue "Kleinheubacher Berichte 2017". It is a result of the Kleinheubacher Tagung 2017, Miltenberg, Germany, 25-27 September 2017. 
Acknowledgements. We would like to acknowledge funding from the DFG SFB/TRR 196 (Marie). Moreover Jared Gwaro greatly acknowledges the financial support given by the DAAD and Kenyan government.

Edited by: Thomas Kleine-Ostmann

Reviewed by: Thorsten Probst and one anonymous referee

\section{References}

Brenner, C., Hofmann, M., Scheller, M., Shakfa, M. K., Koch, M., Mayorga, I. C., Klehr, A., Erbert, G., and Tränkle, G.: Compact diode-laser-based system for continuous-wave and quasi-time-domain terahertz spectroscopy, Opt. Lett., 35, 3859, https://doi.org/10.1364/OL.35.003859, 2010.

Brown, E. R., Mcintosh, K. A., Smith, F. W., Manfra, M. J., and Dennis, C. L.: Measurement of optical-heterodyne conversion in low-temperature-grown GaAs, Appl. Phys. Lett., 62, 1206-1208, 1993.

Chen, P., Blake, G. A., Gaidis, M. C., Brown, E. R., McIntosh, K. A., Chou, S. Y., Nathan, M. I., and Williamson, F.: Spectroscopic applications and frequency locking of $\mathrm{THz}$ photomixing with distributed-Bragg-reflector diode lasers in lowtemperature-grown GaAs, Appl. Phys. Lett., 71, 1601-1603, https://doi.org/10.1063/1.119845, 1997.

Chi, M., Jensen, O. B., and Petersen, P. M.: High-power dualwavelength external-cavity diode laser based on tapered amplifier with tunable terahertz frequency difference, Opt. Lett., 36, 2626, https://doi.org/10.1364/OL.36.002626, 2011.

Criado, A. R., Acedo, P., Carpintero, G., de Dios, C., and Yvind, $\mathrm{K}$.: Observation of phase noise reduction in photonically synthesized sub-THz signals using a passively mode-locked laser diode and highly selective optical filtering, Opt. Express, 20, 1253, https://doi.org/10.1364/OE.20.001253, 2012.

Demers, J. R., Logan Jr., R. T., Bergeron, N. J., and Brown, E. R.: A coherent frequency-domain $\mathrm{THz}$ spectrometer with a signal-tonoise ratio of $60 \mathrm{~dB}$ at $1 \mathrm{THz}$, SPIE Defence and Security Symposium, 6949, 694909, https://doi.org/10.1117/12.777457, 2008.

Deninger, A. J., Göbel, T., Schönherr, D., Kinder, T., Roggenbuck, A., Köberle, M., Lison, F., Müller-Wirts, T., and Meissner, P.: Precisely tunable continuous-wave terahertz source with interferometric frequency control, Rev. Sci. Instrum., 79, 044702, https://doi.org/10.1063/1.2905033, 2008.

Döpke, B., Pilny, R. H., Brenner, C., Klehr, A., Erbert, G., Tränkle, G., Balzer, J. C., and Hofmann, M. R.: Self-optimizing femtosecond semiconductor laser, Opt. Express, 23, 9710, https://doi.org/10.1364/OE.23.009710, 2015.

Fice, M., Rouvalis, E., Ponnampalam, L., Renaud, C., and Seeds, A.: Telecommunications technology-based terahertz sources, Electron. Lett., 46, S28, https://doi.org/10.1049/el.2010.1917, 2010.

Först, M., Janke, C., Nagel, M., Kurz, H., and Bartels, A.: Asynchronous optical sampling for high-speed characterization of integrated resonant $\mathrm{THz}$ sensor arrays, Conference on Lasers and Electro-Optics Europe - Technical Digest, 30, 1405, https://doi.org/10.1109/CLEOE.2005.1568161, 2005.

Friedrich, C. S., Brenner, C., Hoffmann, S., Schmitz, A., Mayorga, I. C., Klehr, A., Erbert, G., and Hofmann, M. R.:
New two-color laser concepts for THz generation, IEEE Journal on Selected Topics in Quantum Electronics, 14, 270-276, https://doi.org/10.1109/JSTQE.2007.912754, 2008.

Friederich, F., Schuricht, G., Deninger, A., Lison, F., Spickermann, G., Haring Bolívar, P., and Roskos, H. G.: Phase-locking of the beat signal of two distributed-feedback diode lasers to oscillators working in the MHz to THz range, Opt. Express, 18, 8621-8629, https://doi.org/10.1364/OE.18.008621, 2010.

Göbel, T., Schoenherr, D., Sydlo, C., Feiginov, M., Meissner, P., and Hartnagel, H.: Continuous-wave terahertz system with electro-optical terahertz phase control, Electron. Lett., 44, 863, https://doi.org/10.1049/el:20081360, 2008.

Göbel, T., Stanze, D., Troppenz, U., Kreissl, J., Sartorius, B., and Schell, M.: Integrated continuous-wave $\mathrm{THz}$ control unit with $1 \mathrm{THz}$ tuning range, in: International Conference on Infrared, Millimeter, and Terahertz Waves, IRMMW-THz, IEEE, 1-3, https://doi.org/10.1109/IRMMW-THz.2012.6380405, 2012.

Göbel, T., Stanze, D., Globisch, B., Dietz, R. J. B., Roehle, H., and Schell, M.: Telecom technology based continuous wave terahertz photomixing system with 105 decibel signal-tonoise ratio and 35 terahertz bandwidth, Opt. Lett., 38, 4197, https://doi.org/10.1364/OL.38.004197, 2013.

Gregory, I. S., Tribe, W. R., Evans, M. J., Drysdale, T. D., Cumming, D. R. S., and Missous, M.: Multi-channel homodyne detection of continuous-wave terahertz radiation, Appl. Phys. Lett., 87, 034106, https://doi.org/10.1063/1.1990249, 2005.

Gwaro, J. O., Brenner, C., Sumpf, B., Klehr, A., Fricke, J., and Hofmann, M. R.: Terahertz frequency generation with monolithically integrated dual wavelength distributed Bragg reflector semiconductor laser diode, IET Optoelectron., 11, 49-52, https://doi.org/10.1049/iet-opt.2016.0054, 2017.

Hermelo, M. F., Shih, P.-T. B., Steeg, M., Ng'oma, A., and Stöhr, A.: Spectral efficient 64-QAM-OFDM terahertz communication link, Opt. Express, 25, 19360, https://doi.org/10.1364/OE.25.019360, 2017.

Hisatake, S., Kim, J. Y., Ajito, K., and Nagatsuma, T.: Self-heterodyne spectrometer using uni-traveling-carrier photodiodes for terahertz-wave generators and optoelectronic mixers, J. Lightwave Technol., 32, 3683-3689, https://doi.org/10.1109/JLT.2014.2321004, 2014.

Hochrein, T., Wilk, R., Mei, M., Holzwarth, R., Krumbholz, N., and Koch, M.: Optical sampling by laser cavity tuning, Opt. Express, 18, 1613, https://doi.org/10.1364/OE.18.001613, 2010.

Hoffmann, S., Luo, X., and Hofmann, M.: Bandwidth limitations of two-colour diode lasers for direct terahertz emission, Electron. Lett., 42, 696, https://doi.org/10.1049/el:20061246, 2006.

Hübers, H. W., Kimmitt, M. F., Hiromoto, N., and Bründermann, E.: Terahertz spectroscopy: System and sensitivity considerations, IEEE T. THz Sci. Techn., 1, 321-331, https://doi.org/10.1109/TTHZ.2011.2159877, 2011.

Jepsen, P. U., Cooke, D. G., and Koch, M.: Terahertz spectroscopy and imaging - Modern techniques and applications, Laser and Photonics Reviews, 5, 124-166, https://doi.org/10.1002/lpor.201000011, 2011.

Jördens, C., Schlauch, T., Li, M., Hofmann, M. R., Bieler, M., and Koch, M.: All-semiconductor laser driven terahertz time-domain spectrometer, Appl. Phys. B, 93, 515-520, https://doi.org/10.1007/s00340-008-3210-4, 2008. 
Kim, Y. and Yee, D.-S.: High-speed terahertz time-domain spectroscopy based on electronically controlled optical sampling, Opt. Lett., 35, 3715, https://doi.org/10.1364/OL.35.003715, 2010.

Kitahara, K., Oto, K., Nakajima, M., and Muro, K.: Note: Development of a high resolution and wide band terahertz spectrometer based on a $1 \mu \mathrm{m}$-band external cavity diode laser, Rev. Sci. Instrum., 84, 126102, https://doi.org/10.1063/1.4842275, 2013.

Klehr, A., Fricke, J., Knauer, A., Erbert, G., Walther, M., Wilk, R., Mikulics, M., and Koch, M.: High-power monolithic two-mode DFB laser diodes for the generation of $\mathrm{THz}$ radiation, IEEE J. Sel. Top. Quant., 14, 289-294, https://doi.org/10.1109/JSTQE.2007.913119, 2008.

Kleine-Ostmann, T., Knobloch, P., Koch, M., Hoffmann, S., Breede, M., Hofmann, M., Hein, G., Pierz, K., Sperling, M., and Donhuijsen, K.: Continuous-wave THz imaging, Electron. Lett., 37, 1461, https://doi.org/10.1049/el:20011003, 2001.

Kohlhaas, R. B., Rehn, A., Nellen, S., Koch, M., Schell, M., Dietz, R. J. B., and Balzer, J. C.: Terahertz quasi time-domain spectroscopy based on telecom technology for $1550 \mathrm{~nm}$, Opt. Express, 25, 12851, https://doi.org/10.1364/OE.25.012851, 2017.

Matsuura, S., Tani, M., Abe, H., Sakai, K., Ozeki, H., and Saito, S.: High-resolution terahertz spectroscopy by a compact radiation source based on photomixing with diode lasers in a photoconductive antenna, J. Mol. Spectrosc., 187, 97-101, https://doi.org/10.1006/jmsp.1997.7486, 1998.

Matus, M., Kolesik, M., Moloney, J. V., Hofmann, M., and Koch, S. W.: Dynamics of two-color laser systems with spectrally filtered feedback, J. Opt. Soc. Am. B, 21, 1758, https://doi.org/10.1364/JOSAB.21.001758, 2004.

McIntosh, K. A., Brown, E. R., Nichols, K. B., McMahon, O. B., Dinatale, W. F., and Lyszczarz, T. M.: Terahertz photomixing with diode lasers in low-temperature-grown GaAs, Appl. Phys. Lett., 67, 3844, https://doi.org/10.1063/1.115292, 1995.

Merghem, K., Busch, S. F., Lelarge, F., Koch, M., Ramdane, A., and Balzer, J. C.: Terahertz Time-Domain Spectroscopy System Driven by a Monolithic Semiconductor Laser, J. Infrared, Millim. Te., 38, 958-962, https://doi.org/10.1007/s10762-017-04012, 2017.

Moon, K., Kim, N., Shin, J.-H., Yoon, Y.-J., Han, S.-P., and Park, K. H.: Continuous-wave terahertz system based on a dual-mode laser for real-time non-contact measurement of thickness and conductivity, Opt. Express, 22, 2259, https://doi.org/10.1364/OE.22.002259, 2014.

Morikawa, O., Tonouchi, M., and Hangyo, M.: Sub-THz spectroscopic system using a multimode laser diode and photoconductive antenna, Appl. Phys. Lett., 75, 3772-3774, https://doi.org/10.1063/1.125451, 1999.

Morikawa, O., Fujita, M., and Hangyo, M.: Improvement of signalto-noise ratio of a subterahertz spectrometer using a continuouswave multimode laser diode by single-mode fiber optics, Appl. Phys. Lett., 85, 881-883, https://doi.org/10.1063/1.1777800, 2004.

Naftaly, M., Stone, M. R., Malcoci, A., Miles, R. E., and Camara Mayorga, I.: Generation of CW terahertz radiation using twocolour laser with Fabry-Perot etalon, Electron. Lett., 41, 128, https://doi.org/10.1049/el:20057532, 2005.

O'Brien, S., Osborne, S., Bitauld, D., Brandonisio, N., Amann, A., Phelan, R., Kelly, B., and O'Gorman, J.: Optical synthe- sis of terahertz and millimeter-wave frequencies with discrete mode diode lasers, IEEE T. Microw. Theory, 58, 3083-3087, https://doi.org/10.1109/TMTT.2010.2074931, 2010.

Osborne, S., O’Brien, S., O'Reilly, E., Huggard, P., and Ellison, B.: Generation of $\mathrm{CW} 0.5 \mathrm{THz}$ radiation by photomixing the output of a two-colour 1.49 [micro sign]m Fabry-Perot diode laser, Electron. Lett., 44, 296, https://doi.org/10.1049/el:20083534, 2008.

Paquet, R., Blin, S., Myara, M., Gratiet, L. L., Sellahi, M., Chomet, B., Beaudoin, G., Sagnes, I., and Garnache, A.: Coherent continuous-wave dual-frequency high-Q external-cavity semiconductor laser for $\mathrm{GHz}-\mathrm{THz}$ applications, Opt. Lett., 41, 3751, https://doi.org/10.1364/OL.41.003751, 2016.

Park, I., Sydlo, C., Fischer, I., Elsäßer, W., and Hartnagel, H. L.: Generation and spectroscopic application of tunable continuouswave terahertz radiation using a dual-mode semiconductor laser, Meas. Sci. Technol., 19, 065305, https://doi.org/10.1088/09570233/19/6/065305, 2008.

Preu, S., Döhler, G. H., Malzer, S., Stöhr, A., Rymanov, V., Göbel, T., Brown, E. R., Feiginov, M., Gonzalo, R., Beruete, M., and Navarro-Cía, M.: Principles of THz Generation, in: Semiconductor THz Technology: Devices and Systems at Room Temperature Operation, 3-68, John Wiley \& Sons, Ltd, Chichester, UK, https://doi.org/10.1002/9781118920411.ch2, 2015.

Probst, T., Rehn, A., and Koch, M.: Compact and lowcost THz QTDS system, Opt. Express, 23, 21972, https://doi.org/10.1364/OE.23.021972, 2015.

Roggenbuck, A., Schmitz, H., Deninger, A., Cámara Mayorga, I. C., Hemberger, J., Güsten, R., and Grüninger, M.: Coherent broadband continuous-wave terahertz spectroscopy on solid-state samples, New J. Phys., 12, 043017, https://doi.org/10.1088/13672630/12/4/043017, 2010.

Rouvalis, E., Fice, M. J., Renaud, C. C., and Seeds, A. J.: Optoelectronic detection of millimetre-wave signals with travellingwave uni-travelling carrier photodiodes, Opt. Express, 19, 2079, https://doi.org/10.1364/OE.19.002079, 2011.

Rymanov, V., Babiel, S., Wachholz, M., Dulme, S., and Stöhr, A.: Ultra-broadband integrated photonic $200-300 \mathrm{GHz}$ transmitters for wireless radio-over-fiber applications, in: International Conference on Infrared, Millimeter, and Terahertz Waves, IRMMW-THz, 1-2, IEEE, https://doi.org/10.1109/IRMMWTHz.2013.6665453, 2013.

Ryu, H.-C., Kim, N., Han, S.-P., Ko, H., Park, J.-W., Moon, K., and Park, K. H.: Simple and cost-effective thickness measurement terahertz system based on a compact $155 \mu \mathrm{m}$ $\lambda / 4$ phase-shifted dual-mode laser, Opt. Express, 20, 25990, https://doi.org/10.1364/OE.20.025990, 2012.

Sartorius, B., Stanze, D., Göbel, T., Schmidt, D., and Schell, M.: Continuous wave terahertz systems based on $1.5 \mu \mathrm{m}$ telecom technologies, J. Infrared Millim. Te., 33, 405-417, https://doi.org/10.1007/s10762-011-9849-7, 2012.

Sato, K.: Optical pulse generation using fabry-perot lasers under continuous-wave operation, IEEE J. Sel. Top. Quant., 9, 12881293, https://doi.org/10.1109/JSTQE.2003.819503, 2003.

Scheller, M. and Koch, M.: Terahertz quasi time domain spectroscopy, Opt. Express, 17, 17723, https://doi.org/10.1364/OE.17.017723, 2009.

Shibuya, K., Tani, M., and Hangyo, M.: Compact and inexpensive continuous-wave sub- $\mathrm{THz}$ imaging system using a fibercoupled multimode laser diode, IRMMW-THz 2006 - 31st In- 
ternational Conference on Infrared and Millimeter Waves and 14th International Conference on Terahertz Electronics, 90, 428, https://doi.org/10.1109/ICIMW.2006.368636, 2006.

Song, H., Hwang, S., An, H., Song, H.-J., and Song, J.-I.: Continuous-wave $\mathrm{THz}$ vector imaging system utilizing two-tone signal generation and self-mixing detection, Opt. Express, 25, 20718, https://doi.org/10.1364/OE.25.020718, 2017.

Stanze, D., Deninger, A., Roggenbuck, A., Schindler, S., Schlak, M., and Sartorius, B.: Compact cw terahertz spectrometer pumped at $1.5 \mu \mathrm{m}$ wavelength, J. Infrared Millim. Te., 32, 225232, https://doi.org/10.1007/s10762-010-9751-8, 2011.

Tanabe, T., Ragam, S., and Oyama, Y.: Continuous wave terahertz wave spectrometer based on diode laser pumping: Potential applications in high resolution spectroscopy, Rev. Sci. Instrum., 80, 113105, https://doi.org/10.1063/1.3258202, 2009.

Tani, M., Gu, P., Hyodo, M., Sakai, K., and Hidaka, T.: Generation of coherent terahertz radiation by photomixing of dual-mode lasers, Opt. Quant. Electron., 32, 503-520, https://doi.org/10.1023/A:1007070931314, 2000.

Tani, M., Morikawa, O., Matsuura, S., and Hangyo, M.: Generation of terahertz radiation by photomixing with dual- and multiple-mode lasers, Semicond. Sci. Tech., 20, S151-S163, https://doi.org/10.1088/0268-1242/20/7/005, 2005.
Tauser, F., Rausch, C., Posthumus, J. H., and Lison, F.: Electronically controlled optical sampling using $100 \mathrm{MHz}$ repetition rate fiber lasers, in: Commercial and Biomedical Applications of Ultrafast Lasers VIII, edited by: Neev, J., Nolte, S., Heisterkamp, A., and Schaffer, C. B., Vol. 6881, 68810O, https://doi.org/10.1117/12.764506, 2008.

Theurer, M., Göbel, T., Stanze, D., Troppenz, U., Soares, F., Grote, N., and Schell, M.: Photonic-integrated circuit for continuous-wave $\mathrm{THz}$ generation, Opt. Lett., 38, 3724, https://doi.org/10.1364/OL.38.003724, 2013.

Thirunavukkuarasu, K., Langenbach, M., Roggenbuck, A., Vidal, E., Schmitz, H., Hemberger, J., and Grüninger, M.: Selfnormalizing phase measurement in multimode terahertz spectroscopy based on photomixing of three lasers, Appl. Phys. Lett., 106, 031111, https://doi.org/10.1063/1.4906374, 2015.

Verghese, S., McIntosh, K. A., Calawa, S., Dinatale, W. F., Duerr, E. K., and Molvar, K. A.: Generation and detection of coherent terahertz waves using two photomixers, Appl. Phys. Lett., 73, 3824-3826, https://doi.org/10.1063/1.122906, 1998.

Wang, C. L. and Pan, C. L.: Tunable dual-wavelength operation of a diode array with an external grating-loaded cavity, Appl. Phys. Lett., 64, 3089-3091, https://doi.org/10.1063/1.111356, 1994. 